\title{
Editorial
}

\section{Bacteremia and Probiotics: A Novel Culture-Independent Analytical Method Evolves Disease Concepts}

\author{
Yuichiro Yamashiro ${ }^{a}$ Koji Nomoto ${ }^{b}$ \\ a Probiotics Research Laboratory, Juntendo University Graduate School of Medicine, and ${ }^{\mathrm{b}}$ Division of Molecular \\ Microbiology, Tokyo University of Agriculture, Tokyo, Japan
}

The term "bacteremia" is defined as the presence of bacteria in the bloodstream; it evidenced by a positive blood culture. Once in circulation, bacteria spread extensively in the body. They reach and colonize the tissues, particularly suitable for their multiplication.

In the body, most disease-causing bacteria first attach or adhere to the host cells, usually the epithelial cells. After the bacteria have established a primary site of infection, they multiply and spread directly through tissues or via lymphatic system into the bloodstream (bacteremia). The systemic response to any class of microorganism can be harmful. Living bodies develop both local and systemic responses to the invading bacteria, which may elicit fever or hypothermia, leukocytosis or leukopenia, tachycardia or tachypnea. In the presence of an infection, these can be denoted as the cardinal signs of sepsis [1]. Sepsis is a leading cause of morbidity and death among hospitalized patients. The disease process results from a complex interplay of host immune responses and infectious bacteria (Fig. 1), and in severe cases can progress to multiple organ system dysfunction followed by organ failure and death.
Culture remains the gold standard for the diagnosis of bacteremia in everyday clinical practice, but its clinical utility is limited by slow turnaround times and low numbers of bacteria in patient's blood. Therefore, nowadays, various novel methods based on the specific identification of microbial DNA or RNA in blood or tissue samples are being explored and sought after to be introduced and integrated into the workflows of modern diagnostic laboratories. One such example is the YIF-SCAN ${ }^{\circledR}$ analytical system [2] which is based on the reverse-transcription quantitative PCR assays targeting bacterial 16S rRNA "molecules". The method utilizes a wide array of highly specific primers and, unlike DNA-based methods, provides highly sensitive quantification of 'live' bacteria in both blood and fecal samples. The minimum detectable number of the targeted bacteria by using this method is 1 bacterial cell per $0.5-1 \mathrm{ml}$ of human peripheral blood $[3,4]$. Therefore, owing to its high analytical sensitivity, this method has made a great advancement to investigate Koch's postulate [5]. Some pathogens are too difficult or even impossible to be diagnosed and applied to the Koch's postulate to establish them as the cause of associ- 


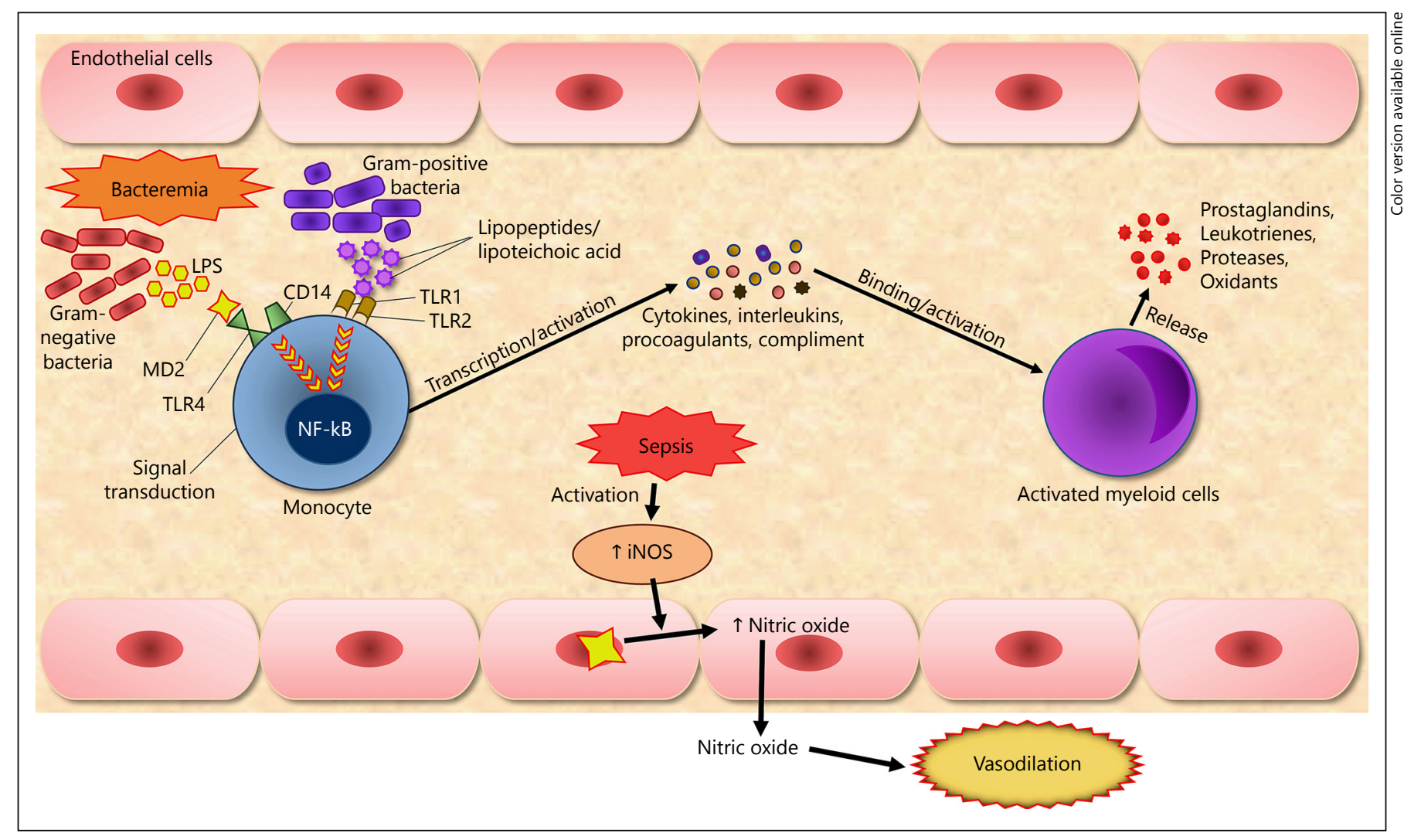

Fig. 1. Immunopathogenesis of sepsis following invasive infection (bacteremia). In the case of invasive infection, microbial pathogens after entering into the host tissue are first sensed by myeloid cells of the innate immune system through binding to the patternrecognition receptors such as TLRs present on the cell surface and in endosomal compartments. CD, cluster determinant; iNOS, cytokine-inducible nitric-oxide synthase; LPS, lipopolysaccharide; MD2, myeloid differentiation factor 2; NF-kB, nuclear factor kappa-light-chain-enhancer of activated B-cells; TLR, toll-like receptors. ated diseases. Furthermore, the recent and 'unexpected' findings of bacteremia, as proved by the quantitative PCR analysis, may also support in evolving the disease concepts.

The link between the altered gut bacterial composition and the development of obesity and metabolic syndrome such as type 2 diabetes mellitus in humans is becoming clear, and these disease states are characterized by lowgrade inflammation, insulin resistance, and increased gut permeability with high leakage of plasma lipopolysaccharide (LPS) and LPS binding protein from gut into the circulation, as the gut microbiota is a rich source of LPS (an endotoxin and a major component of the outer membrane of gram-negative bacteria) [6].

In these circumstances, bacterial translocation of intestinal commensal bacteria into the adipose tissue and the blood is suggested and has been demonstrated in diabetic mice [7] and in humans [8] without obvious inflammatory symptoms in general. From the surgical aspect, the high incidence of bacteremia originating from the intestine during the surgery of abdominal cavity [2] could probably be thought as the cause of postoperative ailments, for example, fever and/or sepsis, thereby clearly underscoring the need to reconsider the management strategies. Fortunately, probiotics treatment has been found to prevent and/or ameliorate the bacteremia in such surgical cases $[2,4]$ and also in diabetic mice [7].

The findings mentioned above evolve our understanding of the disease concepts and can be envisioned to offer new diagnostic and therapeutic targets and avenues. In these contexts, the purpose of this special supplement issue is to provide unbiased updated information on bacteremia and probiotics, a rapidly progressing field in clinical medicine, and to contribute significantly to our knowledge and actions in this discipline. 


\section{References}

1 Munford RS: Severe sepsis and septic shock; in Harison's Principles of Internal Medicine; in Kasper D, Fauci, A, Hauser S, Longo D, Jameson JL, Loscalzo J, Anthony SF, Stephen LH (eds). ed 19th, McGraw Hill Education, USA, 2015, pp 1751-1756.

2 Matsuda K, Tsuji H, Asahara T, et al: Sensitive quantitative detection of commensal bacteria by rRNA-targeted reverse transcription-PCR. Appl Environ Microbiol 2007;73: 32-39.

3 Mizuno T, Yokoyama Y, Nishio H, et al: Intraoperative bacterial translocation detected by bacterium-specific ribosomal RNA-tar- geted reverse-transcriptase polymerase chain reaction for the mesenteric lymph node strongly predicts postoperative infectious complications after major hepatectomy for biliary malignancies. Ann Surg 2010;252: 1013-1019.

4 Okazaki T, Asahara T, Yamataka A, et al: Intestinal microbiota in pediatric surgical cases administered bifidobacterium breve: a Randomized Controlled Trial. J Pediatr Gastroenterol Nutr 2016;63:46-50.

5 Hess M: Commensal or pathogen - a challenge to fulfil Koch's Postulates. Br Poult Sci 2017;58:1-12.
6 Cani PD, Amar J, Iglesias MA, et al: Metabolic endotoxemia initiates obesity and insulin resistance. Diabetes 2007;56:17611772.

7 Amar J, Chabo C, Waget A, et al: Intestinal mucosal adherence and translocation of commensal bacteria at the early onset of type 2 diabetes: molecular mechanisms and probiotic treatment. EMBO Mol Med 2011;3:559572.

8 Sato J, Kanazawa A, Ikeda F, et al: Gut dysbiosis and detection of "live gut bacteria" in blood of Japanese patients with type 2 diabetes. Diabetes Care 2014;37:2343-2350. 